\title{
SHARKOVSKII'S ORDER AND THE STABILITY OF PERIODIC POINTS OF MAPS OF THE INTERVAL
}

\author{
GUANG YUAN ZHANG and QING ZHONG LI
}

(Received 12 May 1998; revised 9 August 1999)

Communicated by A. Hillman

\begin{abstract}
Let $f$ be a $C^{r}(r \geq 0)$ map from the interval $[0,1]$ into itself and $m$ be a positive integer. This paper gives a sufficient and necessary condition under which the set of periodic points of period $\boldsymbol{m}$ disappears after a certain small $C^{r}$-perturbation.
\end{abstract}

1991 Mathematics subject classification (Amer. Math. Soc.): primary 58F20.

\section{Introduction}

In this paper, we denote by $I$ the interval $[0,1]$, by $C^{0}(I, I)$ the space of all continuous maps from $I$ into $I$, by $\mathbb{N}$ the set of positive integers and by $\triangleleft$ the Sharkovskii's order of $\mathbb{N}$ :

\begin{tabular}{|c|c|c|c|c|}
\hline & $3 \triangleleft$ & $5 \triangleleft \cdots \triangleleft$ & $2 k+1 \triangleleft$ & $2 k+3 \triangleleft \cdots$ \\
\hline$\cdots \triangleleft$ & $2 \cdot 3 \triangleleft$ & $2.5 \triangleleft \cdots \triangleleft$ & $2(2 k+1) \triangleleft$ & $2(2 k+3) \triangleleft \cdots$ \\
\hline$\cdots \triangleleft$ & $2^{2} 3 \triangleleft$ & $2^{2} 5 \triangleleft \cdots \triangleleft$ & $2^{2}(2 k+1) \triangleleft$ & $2^{2}(2 k+3) \triangleleft \cdots$ \\
\hline & & $\cdots$ & $\cdots$ & \\
\hline$\cdots \triangleleft$ & $2^{n} 3 \triangleleft$ & $2^{n} 5 \triangleleft \cdots \triangleleft$ & $2^{n}(2 k+1) \triangleleft$ & $2^{n}(2 k+3) \triangleleft$ \\
\hline
\end{tabular}

For $f \in C^{0}(I, I)$ and $k \in \mathbb{N}, f^{k}$ is the $k$-th iterate of $f$ defined as $f^{0}=$ id, $f^{1}=f, \ldots, f^{k}=f \circ f^{k-1}$ successively, where id is the identity. For $p \in I$, if

(C) 2000 Australian Mathematical Society $0263-6115 / 2000 \$ A 2.00+0.00$ 
$f^{k}(p)=p$ but $f^{i}(p) \neq p$ for any positive integer $i<k$, then $p$ is called a periodic point of period $k$ or $k$-periodic point of $f$. We denote by $P(f, k)$ the set of all $k$ periodic points of $f$ and by $P P(f)$ the set $\{n \in \mathbb{N} ; f$ has an $n$-periodic point $\}$. One of the most striking results in the theory of one dimensional dynamical systems is Sharkovskii's theorem.

THEOREM 1.1. Let $f \in C^{0}(I, I)$. If $m \in P P(f)$ and $m \triangleleft n$, then $n \in P P(f)$.

This theorem, however, gives no information about the stability of periodic points. On this problem, Block [B] obtained a remarkable result.

THEOREM 1.2. Let $f \in C^{0}(I, I)$ and $m \in P P(f)$. Then there is a positive number $\varepsilon_{0}$ such that any map $g \in C^{0}(I, I)$ with $\max _{x \in I}|g(x)-f(x)|<\varepsilon_{0}$ has an $n$-periodic point if $m \triangleleft n$.

The case $n=m$ is not involved in Block's theorem. In fact, the conclusion no longer holds in this case. If $f_{r}(x)=1-r x, x \in[0,1], r \in[0,1]$, for example, then $P P\left(f_{1}\right)=\{1,2\}$ but $P P\left(f_{r}\right)=\{1\}$ for $r \neq 1$ though $f_{r}$ tends to $f_{1}$ on $[0,1]$ uniformly as $r \rightarrow 1$.

Now, let $m \in \mathbb{N}, r \in\{0, \infty\} \cup \mathbb{N}$ and $f \in C^{r}(I, I)$. Suppose that $P(f, m) \neq \emptyset$ and let $J$ be a component of $P(f, m)$. If there exist a sequence $\left\{f_{n}\right\} \subset C^{r}(I, I)$ and a relatively open subset $V$ of $I$ containing $J$ such that $f_{n} \stackrel{C^{\prime}}{\rightarrow} f$ as $n \rightarrow \infty$ and $V \cap P\left(f_{n}, m\right)=\emptyset$ for all $n$, then we say that $J$ is removable under $C^{r}$-perturbation. If there exists a sequence $\left\{f_{n}\right\} \subset C^{r}(I, I)$ such that $f_{n} \stackrel{C^{r}}{\rightarrow} f$ as $n \rightarrow \infty$ and $P\left(f_{n}, m\right)=\emptyset$ for all $n$, then we say that $P(f, m)$ is removable under $C^{r}$-perturbation. Here $f_{n} \stackrel{C^{r}}{\rightarrow} f$ as $n \rightarrow \infty$ means that, if $r<\infty$, then

$$
\left\|f_{n}-f\right\|_{C^{r}(I)}=\max _{0 \leq i \leq r} \max _{x \in I}\left|f_{n}^{(i)}(x)-f^{(i)}(x)\right| \rightarrow 0 \quad \text { as } n \rightarrow \infty,
$$

and if $r=\infty$, then for each $r^{\prime}<\infty$,

$$
\left\|f_{n}-f\right\|_{C^{\prime}(I)} \rightarrow 0 \text { as } n \rightarrow \infty .
$$

For a subset $J$ of $I$, we always use the term neighbourhood of $J$ to indicate any relatively open subset of $I$ containing $J$. This paper is to give a condition under which the set of periodic points of a certain period is removable. The main results of this paper are the following theorems.

THEOREM 1.3. Let $m \in \mathbb{N}, r \in\{0, \infty\} \cup \mathbb{N}, f \in C^{r}(I, I)$ and let $J$ be a component of $P(f, m)$. Then $J$ is removable under $C^{r}$-perturbation if and only if: $J \neq[0,1]$ and $J$ has a neighborhood $V_{J}$ such that either $f^{m}(x)-x \geq 0$ on $V_{J}$, or $f^{m}(x)-x \leq 0$ on $V_{J}$. Moreover, $f^{m}(x)-x \geq 0$ on $V_{J}$ if $0 \in J$ and $f^{m}(x)-x \leq 0$ on $V_{J}$ if $1 \in J$. 
It follows from this theorem that $J$ is removable under $C^{r}$-perturbation if and only if $J$ is removable under $C^{0}$-perturbation.

THEOREM 1.4. Let $m \in \mathbb{N}, r \in\{0, \infty\} \cup \mathbb{N}$ and $f \in C^{r}(I, I)$. Then $P(f, m)$ is removable under $C^{r}$-perturbation if and only if every component of $P(f, m)$ is removable under $C^{0}$-perturbation.

By Theorem 1.3 and Theorem 1.4, it is reasonable to replace the term removable under $C^{r}$-perturbation by the term removable.

\section{Preparations}

For simplicity, we denote by $\langle a, b\rangle$ any interval with endpoints $a$ and $b$ ( $a$ may be larger than $b$ ).

LEMMA 2.1. Let $f \in C^{0}(I, I)$ and $J$ be a component of $P(f, m)$. Then $J, f(J)$, $\ldots, f^{m-1}(J)$ are pairwise disjoint components of $P(f, m)$.

PROOF. It is clear that $J, f(J), \ldots, f^{m-1}(J)$ are components of $P(f, m)$ and $J=f^{m}(J)$. If $f^{m_{1}}(J) \cap f^{m_{2}}(J) \neq \emptyset$ for some non-negative integers $m_{1}$ and $m_{2}$ with $m_{1}<m_{2}<m$, then $J \cap f^{m_{2}-m_{1}}(J) \neq \emptyset$, and then $J=f^{m_{2}-m_{1}}(J)$, which implies that there is a point $p \in J$ such that $f^{m_{2}-m_{1}}(p)=p$. This contradicts $p \in J \subset P(f, m)$.

LEMMA 2.2. Let $f \in C^{0}(I, I)$ and $J=\langle a, c\rangle(a \neq c)$ be a component of $P(f, m)$. If $c \notin P(f, m)$ then $m$ must be even and

(a) $c \in P(f, m / 2)$;

(b) $a \in P(f, m)$; and

(c) there is another component $J^{*}=\langle b, c\rangle$ of $P(f, m)$ such that $b \in P(f, m)$, $J^{*} \cap J=\emptyset, f^{m / 2}\left(J^{*}\right)=J$ and $f^{m / 2}(J)=J^{*}$.

ProOF. Clearly $f^{m}(c)=c$, and then $c$ is a periodic point of period $m^{\prime}, m^{\prime}$ is some positive integer with $m^{\prime}<m$ and $m^{\prime} \mid m$. By Lemma $2.1, J^{*}=f^{m^{\prime}}(J)$ is another component of $P(f, m)$ which does not join $J$, but $J$ and $J^{*}$ have the same endpoint $c$ since $f^{m^{\prime}}(c)=c$, and then we may assume $J^{*}=\langle b, c\rangle$. On the other hand, it follows from Lemma 2.1 again that $f^{m^{\prime}}\left(J^{*}\right) \cap J^{*}=\emptyset$ and $f^{m^{\prime}}\left(J^{*}\right)$ is a component of $P(f, m)$. Thus $f^{m^{\prime}}\left(J^{*}\right)=J$, which implies $f^{2 m^{\prime}}(J)=J$ and $m^{\prime}=m / 2$, and then (a) is proved.

If $a \notin P(f, m)$, then we have $a \in P(f, m / 2)$ again. Thus, considering that $f^{m / 2}$ is one-to-one on $J$, we have $f^{m / 2}(J)=J$, which is a contradiction. Thus $a \in P(f, m)$ and, for the same reason, $b \in P(f, m)$. Hence (b) and (c) hold. 
LEMMA 2.3. Let $f \in C^{0}(I, I)$, and $J$ be a component of $P(f, m)$. If $a$ is an endpoint of $J$ and $a \in P(f, m) \cap(0,1)$, then there is a sequence $\left\{a_{n}\right\} \subset 1$ tending to $a$ such that $f^{m}\left(a_{n}\right) \neq a_{n}, n=1,2, \ldots$.

PROOF. Without loss of generality, assume $J=[a, b]$ and $a \in(0,1)$. Since $a \in P(f, m)$, the points $a, f(a), \ldots, f^{m-1}(a)$ are pairwise distinct. Thus every point $x$ near $a$ is either an $m$-periodic point of $f$ or $f^{m}(x) \neq x$. But $J$ is a component of $P(f, m)$, and then for every $n \in \mathbb{N}$, there exists a point $a_{n} \in(a-1 / n, a)$ such that $f^{m}\left(a_{n}\right) \neq a_{n}$. This completes the proof.

LEMMA 2.4. Let $a$ and $b$ be real numbers with $a<b$. Then there exists a sequence $\left\{h_{n}\right\} \subset C^{\infty}(R, I)$ such that

(a) $h_{n}(x) \equiv 0$ for $x \in R \backslash(a, b)$ but $h_{n}(x)>0$ on $(a, b)$;

(b) $a<x+h_{n}(x)<b$ for $x \in(a, b)$; and

(c) $\left\|h_{n}\right\|_{C^{n}(I)}=\left\|h_{n}\right\|_{C^{n}([a, b])}<1 / n$.

PROOF. Without loss of generality, assume $[a, b]=[-1,1]$. Let

$$
h_{n}(x)= \begin{cases}\frac{\lambda_{n}}{2} \exp \left\{\frac{1}{x^{2}-1}\right\}, & |x|<1, \\ 0, & |x| \geq 1,\end{cases}
$$

where

$$
\lambda_{n}=\left(n\left\|\exp \left\{\frac{1}{x^{2}-1}\right\}\right\|_{C^{n}([-1,1])}\right)^{-1} .
$$

Then $h_{n}$ has the desired properties.

LEMMA 2.5. Let $T$ be a topological space. If $T$ has a countable basis, then any open covering of $T$ has a countable subcovering [D].

\section{Proofs of Theorem 1.3 and Theorem 1.4}

By Lemma 2.2, it is easy to see that the sufficient and necessary condition in Theorem 1.3 is equivalent to the following condition (i) or condition (ii):

(i) $J$ is a semi-open interval and has a neighborhood $V_{J}$ such that either $f^{m}(x)-$ $x \geq 0$ on $V_{J}$, or $f^{m}(x)-x \leq 0$ on $V_{J}$;

(ii) $J$ is a closed interval, $J \neq[0,1]$ and has a neighborhood $V_{J}$ such that either $f^{m}(x)-x \geq 0$ on $V_{J}$, or $f^{m}(x)-x \leq 0$ on $V_{J}$; moreover, if $0 \in J$ then $f^{m}(x)-x \geq 0$ on $V_{J}$ and if $1 \in J$ then $f^{m}(x)-x \leq 0$ on $V_{J}$. 
PROOF OF THEOREM 1.3. To prove the necessity, we assume that $J$ is a component of $P(f, m)$ that is removable under $C^{r}$-perturbation and show that $J$ satisfies the above condition (i) or (ii). By Lemma 2.2, $J$ is either a semi-open interval or a closed interval.

If $J=[0,1]$, then $f=$ id. Since any map in $C^{0}(I, I)$ has at least one fixed point, as a component of $P(\mathrm{id}, m), J$ can not be removable under $C^{r}$-perturbation. Thus $J \neq[0,1]$

If $J \neq[0,1]$ and $J$ does not satisfy (i) or (ii), then by Lemma 2.1 and Lemma 2.2 we can find two sequences $\left\{r_{n}\right\}$ and $\left\{r_{n}^{\prime}\right\}$ contained in $[0,1]$, such that each sequence converges to an endpoint of $J$, that $\left(f^{m}\left(r_{n}\right)-r_{n}\right)\left(f^{m}\left(r_{n}^{\prime}\right)-r_{n}^{\prime}\right)<0$ and that $f^{i}\left(\left[r_{n}, r_{n}^{\prime}\right]\right)$, $i=0,1, \ldots, m-1$, are pairwise disjoint. By continuity, for any $n \in \mathbb{N}$ and any $g \in C^{0}(I, I)$, if $\|g-f\|_{C^{0}(I)}$ is small enough, then $g$ has at least one periodic point of period $m$ in $\left(r_{n}, r_{n}^{\prime}\right)$ (note that $f^{l}(x)-x \neq 0$ on $\left[r_{n}, r_{n}^{\prime}\right]$ for $l=1,2, \ldots m-1$ ), that is to say, $J$ cannot be removable under $C^{0}$-perturbation. Thus $J$ cannot be removable under $C^{r}$-perturbation, which is a contradiction.

To prove the sufficiency, we only prove Proposition 3.1 which clearly implies the sufficiency and will be used to prove Theorem 1.4.

PROPOSITION 3.1. Let $m \in \mathbb{N}, r \in\{0, \infty\} \cup \mathbb{N}, f \in C^{r}(I, I)$ and let $J$ be a component of $P(f, m)$ satisfying (i) or (ii). Then there exist a sequence $\left\{h_{n}\right\} \subset C^{\infty}(I, I)$ and a neighbourhood $V_{J}$ of $J$ such that

(a) $\left.h_{n}\right|_{I \backslash V_{J}} \equiv 0,\left\|h_{n}\right\|_{C^{n}(I)}<1 / n$ and $h_{n}+$ id bijectively maps $V_{J}$ onto a subset of $V_{J}$, where id is the identity;

(b) for $f_{n}=f \circ\left(h_{n}+\mathrm{id}\right)$, if $J$ satisfies (i), then $f_{n}^{m}$ has only one fixed point $c$ in $\bar{V}_{J}$, furthermore, $c$ is an $m / 2$-periodic point of both $f_{n}$ and $f$; and if $J$ satisfies (ii), then $f_{n}^{m}$ has no fixed point on $\bar{V}_{J}$; and

(c) $P\left(f_{n}^{m}, 1\right) \subset P\left(f^{m}, 1\right), P\left(f_{n}, m\right) \subset P(f, m)$ and each component $J^{\prime}$ of $P\left(f_{n}, m\right)$ is a component of $P(f, m)$ and satisfies (i) or (ii) for $f_{n}$ if $J^{\prime}$ satisfies (i) or (ii) for $f$.

PROOF. We first assume that $J$ satisfies (i). Without loss of generality, we additionally assume $J=[a, c)$. Thus by Lemma $2.2, f^{m / 2}(c)=c$ and there exists a number $b>c$ such that $J_{0}=(c, b]$ is another component of $P(f, m)$.

If $a \neq 0$, then by Lemma 2.3, there exists a point $a^{\prime} \in[0, a)$ such that $f^{m}\left(a^{\prime}\right)-a^{\prime} \neq 0$ and $\operatorname{sgn}\left\{\left(f^{m}\left(a^{\prime}\right)-a^{\prime}\right)\left(f^{m}(x)-x\right)\right\} \geq 0$, for all $x \in\left[a^{\prime}, c\right]$. Without loss of generality, assume

$$
f^{m}\left(a^{\prime}\right)>a^{\prime} \text { and } f^{m}(x) \geq x, \quad x \in\left[a^{\prime}, c\right] .
$$

Since $a \in P(f, m)$, by Lemma 2.1 , we can chose $a^{\prime}$ such that

$$
\left[a^{\prime}, c\right) \cap f^{i}\left(\left[a^{\prime}, c\right)\right)=\emptyset, \quad \text { for } i=1,2, \ldots, m-1 .
$$


It follows from Lemma 2.4 that there exists a sequence $\left\{h_{n}\right\} \subset C^{\infty}(I, I)$ such that $h_{n}(x)>0 \quad$ for $x \in\left(a^{\prime}, c\right), \quad h_{n}(x) \equiv 0 \quad$ for $x \in I \backslash\left(a^{\prime}, c\right), \quad\left\|h_{n}\right\|_{C^{n}(I)}<1 / n$, and $h_{n}(x)+x$ bijectively maps $\left[a^{\prime}, c\right]$ onto $\left[a^{\prime}, c\right]$.

Setting $f_{n}(x)=f\left(x+h_{n}(x)\right), x \in I$, we have that $f_{n} \in C^{r}(I, I)$,

$$
\begin{aligned}
& f_{n}(x)-\left.f(x)\right|_{I \backslash\left(a^{\prime}, c\right)} \equiv 0, \quad \text { and } \\
& f_{n}\left(\left[a^{\prime}, c\right)\right)=f\left(\left[a^{\prime}, c\right)\right) .
\end{aligned}
$$

Thus by (3.2) we infer that

$$
\left[a^{\prime}, c\right) \cap f_{n}^{i}\left(\left[a^{\prime}, c\right)\right)=\emptyset, \quad i=1,2, \ldots, m-1,
$$

and then by (3.2) and (3.3), $f_{n}^{i}(x)=f^{i}(x)$ for $x \in f\left(\left[a^{\prime}, c\right)\right)\left(=f_{n}([a, c))\right)$ and $i=1,2, \ldots, m-1$, which implies

$$
f_{n}^{m}(x)=f^{m-1}\left(f_{n}(x)\right)=f^{m}\left(x+h_{n}(x)\right), \quad x \in\left[a^{\prime}, c\right) .
$$

Hence it follows from (3.1) and the property of $h_{n}$ that

$$
f_{n}^{m}(x)=f^{m}\left(x+h_{n}(x)\right) \geq x+h_{n}(x)>x \text { for } x \in\left[a^{\prime}, c\right) .
$$

So, $f_{n}^{m}$ has no fixed point in $\left[a^{\prime}, c\right)$.

It follows from (3.2) and (3.3) that $f_{n}$ and $f$ coincide on the set $\{c, f(c), \ldots$, $\left.f^{m / 2-1}(c)\right\}$, and then $c \in P\left(f_{n}, m / 2\right)$.

By Lemma $2.2, f^{m / 2}((c, b))=(a, c)$, and then (3.2), (3.3), and Lemma 2.1 imply

$$
f_{n}^{m / 2}((c, b))=f^{m / 2}((c, b))=(a, c),
$$

which implies that $f_{n}^{m}$ has no fixed point in $(c, b)$ since we have proved that $f_{n}^{m}$ has no fixed point in $\left(a^{\prime}, c\right)(\supset(a, c))$. Thus, putting $V_{J}=\left(a^{\prime},(c+b) / 2\right)$, we obtain (a) and (b), which imply (c).

If $a=0$, the proof is just slightly different. By Lemma 2.4 , for any $n \in \mathbb{N}$ there exists a map $h_{n} \in C^{\infty}(I, I)$ such that

$$
h_{n}(x)>0 \quad \text { for } x \in[0, c), \quad h_{n}(x) \equiv 0 \quad \text { for } x \in[c, 1], \quad\left\|h_{n}\right\|_{C^{n}(I)}<1 / n,
$$

and $h_{n}(x)+x$ bijectively maps $[0, c]$ onto $[h(0), c]$.

Repeating the argument in the case $J=[a, c)$, one can prove that conditions (a)-(c) hold for $\left\{h_{n}\right\}$ and $V_{J}=[0,(c+b) / 2)$.

Now we assume that $J=[a, b]$ is a closed interval and satisfies (ii). If $a \neq 0$ and $b \neq 1$, then by condition (ii), we can assume that there exist a point $a^{\prime} \in[0, a)$ and a point $b^{\prime} \in(b, 1]$ such that

$$
f^{m}(x)-x \geq 0, \quad x \in\left(a^{\prime}, b^{\prime}\right)
$$


Since $J=[a, b]$ is a component of $P(f, m)$, it follows from (3.4), and Lemma 2.1 that $a^{\prime}$ and $b^{\prime}$ can be chosen so that

$$
\left[a^{\prime}, b^{\prime}\right] \cap f^{i}\left(\left[a^{\prime}, b^{\prime}\right]\right)=\emptyset, \quad i=1,2, \ldots, m-1 .
$$

By (3.4) and (3.5), and using similar argument as in the case $J=[a, c)$, we can construct a sequence $\left\{h_{n}\right\} \subset C^{\infty}(I, l)$ such that $V_{J}=\left(a^{\prime}, b^{\prime}\right)$ and $\left\{h_{n}\right\}$ satisfy (a)-(c).

If $a=0$ or $b=0$, similarly to the case of $J=[a, b]$ with $a \neq 0$ and $b \neq 1$ and to the case of $J=[a, c)$, we can prove the existence of $h_{n}$ and $V_{J}$. So (a)-(c) hold in any case. This completes the proof.

PROOF OF THEOREM 1.4. The necessity is obvious.

To prove the sufficiency, suppose that every component of $P(f, m)$ is removable under $C^{0}$-perturbation. Then by Theorem 1.3 , every component of $P(f, m)$ satisfies condition (i) or (ii).

We first assume that $r<\infty$.

By Proposition 3.1, every component $J$ of $P(f, m)$ has a neighbourhood $V_{J}$ such that to any positive number $\varepsilon$ corresponds a $C^{\infty}$-map $h_{\varepsilon} \in C^{\infty}(I, I)$ such that $g_{\varepsilon}=$ $f \circ h_{\varepsilon}$ satisfies the following $\left(V_{J}, f, \varepsilon\right)$-conditions:

(1) $\left.\left(g_{\varepsilon}-f\right)\right|_{\Lambda \backslash V_{j}} \equiv 0$ and $\left\|g_{\varepsilon}-f\right\|_{C^{r}(I)}<\varepsilon$;

(2) $P\left(g_{\varepsilon}, m\right) \subset P(f, m)$ and any component $J^{\prime}$ of $P\left(g_{\varepsilon}, m\right)$ is also a component of $P(f, m)$ and satisfies (i) or (ii) for $g_{\varepsilon}$ and $V_{J^{\prime}}$; and

(3) If $J$ satisfies (i), then $g_{\varepsilon}^{m}$ has only one fixed point $c_{J}$ in $\bar{V}_{J}$, which is an $m / 2$ periodic point of both $g_{\varepsilon}$ and $f$; if $J$ satisfies (ii), then $g_{\varepsilon}^{m}$ has no fixed point in $\bar{V}_{J}$.

The union of all $V_{J} \mathrm{~s}$ is an open covering of $P(f, m)$. Since $I$ has a countable basis, the covering has a countable subcovering according to Lemma 2.5 . Thus there exists a sequence $\left\{J_{i}\right\}$ of components of $P(f, m), i=1,2, \ldots$, such that $\bigcup_{i=1}^{\infty} V_{J_{i}} \supset P(f, m)$.

Fix a positive number $\varepsilon$ arbitrarily and put $\bar{V}_{J}=\left[a_{J}, b_{J}\right]$. Without loss of generality, assume that $J_{i}$ and $V_{J_{i}}$ satisfy condition (i) if $i$ is odd and satisfy condition (ii) if $i$ is even.

For $n \in \mathbb{N}$ and $k \in \mathbb{N}$ let

$$
V_{J_{2 k-1}}^{n}=V_{J_{2 k-1}} \backslash\left(c_{J_{2 k-1}}-1 / n, c_{J_{2 k-1}}+1 / n\right) \quad \text { and } \quad V_{J_{2 k}}^{n}=V_{J_{2 k}} .
$$

Then there exists a map $f_{1} \in C^{r}(I, I)$ satisfying $\left(V_{J_{1}}, f, \varepsilon / 2\right)$-conditions. By continuity, $f_{1}$ also satisfies the following condition $\left(\mathrm{P}_{1}\right)$ :

$\left(\mathrm{P}_{1}\right)$ There exists a positive number $\varepsilon_{1}<\varepsilon / 2$, such that for each $g \in C^{r}(I, I), g^{m}$ has no fuxed point in $V_{J_{1}}^{1}$ if $\left\|f_{1}-g\right\|_{C^{r}(I)}<\varepsilon_{1}$.

For the same reason, there is a map $f_{2} \in C^{r}(I, I)$ satisfying $\left(V_{J_{2}}, f_{1}, \varepsilon_{1} / 2\right)$ conditions (if $f_{1}$ has no $m$-periodic point in $V_{J_{2}}$ then define $f_{2}=f_{1}$ ) and the following condition $\left(\mathrm{P}_{2}\right)$ : 
$\left(\mathrm{P}_{2}\right)$ There exists a positive number $\varepsilon_{2}<\varepsilon_{1} / 2$, such that for each $g \in C^{r}(I, I), g^{m}$ has no fuxed point in $V_{J_{1}}^{2} \cup V_{J_{2}}^{1}$ if $\left\|f_{2}-g\right\|_{C^{r}(I)}<\varepsilon_{2}$.

Now, repeating the same argument, we can successively obtain a sequence $\left\{\varepsilon_{n}\right\}_{n=1}^{\infty}$ of positive numbers and a sequence $\left\{f_{n}\right\}_{n=1}^{\infty} \subset C^{r}(I, I)$ such that $\varepsilon_{n+1}<\varepsilon_{n} / 2$, $\left\|f_{n+1}-f_{n}\right\|_{C^{r}(I)}<\varepsilon_{n} / 2, f_{n+1}$ satisfies $\left(V_{J_{n+1}}, f_{n}, \varepsilon_{n} / 2\right)$-conditions and

$\left(\mathrm{P}_{n}\right)$ For each $g \in C^{r}(I, I), g^{m}$ has no fixed point in $V_{J_{1}}^{n} \cup V_{J_{2}}^{n-1} \cup \cdots \cup V_{J_{n}}^{1}$ if $\left\|f_{n}-g\right\|_{C^{\prime}(l)}<\varepsilon_{n}, n=1,2, \ldots$

Clearly, $f_{n} \stackrel{C^{r}}{\rightarrow} f^{*}$ for some $f^{*} \in C^{r}(I, I)$ and it is easy to see that $\left\|f^{*}-f\right\|_{C^{r}(I)}<\varepsilon$ and $\left\|f^{*}-f_{n}\right\|_{C^{r}(I)}<\varepsilon_{n}, n=1,2, \ldots$ Thus by condition $\left(\mathrm{P}_{n}\right), f^{*}$ has no $m$-periodic point in $V_{J_{1}}^{n} \cup V_{J_{2}}^{n-1} \cup \cdots \cup V_{J_{n}}^{1}$ for every $n \in \mathbb{N}$, which implies that $f^{*}$ has no $m$-periodic point in $\bigcup_{n=1}^{\infty}\left\{V_{J_{1}}^{n} \cup V_{J_{2}}^{n-1} \cup \cdots \cup V_{J_{n}}^{1}\right\}=\left\{\bigcup_{n=1}^{\infty} V_{J_{n}}\right\} \backslash\left\{c_{J_{2 k-1}} \mid k \in \mathbb{N}\right\}$. On the other hand, $\left(V_{J_{n+1}}, f_{n}, \varepsilon_{n} / 2\right)$-conditions, $n=1,2, \ldots$, imply that

$$
\left.\left(f^{*}-f\right)\right|_{I \backslash\left\{\bigcup_{n=1}^{\infty} v_{J_{n}}\right\}} \equiv 0
$$

and that $\left\{c_{J_{2 k-1}} \mid k \in \mathbb{N}\right\} \subset P\left(f^{*}, m / 2\right)$. Hence $f^{*}$ has no periodic point of period $m$ in $I \backslash\left\{\bigcup_{n=1}^{\infty} V_{J_{n}}\right\}$ and $\left\{c_{J_{2 k-1}} \mid k \in \mathbb{N}\right\}$, and then $f^{*}$ has no $m$-periodic point in $I$. Thus the sufficiency is proved in the case $r<\infty$.

If $r=\infty$, then the above $g_{\varepsilon}$ is of class $C^{\infty}$. Thus for each $k \in \mathbb{N}$, repeating the above arguments, we can construct a sequence $\left\{f_{n k}\right\} \subset C^{\infty}(I, I)$ such that $\left\|f-f_{1 k}\right\|_{C^{k}(I)}<$ $1 /(2 k),\left\|f_{n k}-f_{n+1, k}\right\|_{C^{n+k}(I)}<1 /\left(2^{n+1} k\right)$ for each $n, \lim _{n \rightarrow \infty}\left\|f_{n k}-f_{k}^{*}\right\|_{C^{k}(I)}=0$ for some $f_{k}^{*} \in C^{\infty}(I, I)$ with $P\left(f_{k}^{*}, m\right)=\emptyset$. Clearly, $\left\|f-f_{k}^{*}\right\|_{C^{k}(I)}<1 / k$ and then $f_{k}^{*} \stackrel{C^{\infty}}{\rightarrow} f$ as $k \rightarrow \infty$. This completes the proof.

\section{References}

[B] L. Block, 'Stability of the periodic orbits in the theorem of Sharkovskii', Proc. Amer. Math. Soc. 81 (1981), 333-336.

[D] J. Dugundji, Topology (Allyn and Bacon, Boston, 1966).

[S] A. N. Sharkovskii, 'Coexistence of cycles of a continuous map of the line into itself', Ukrain. Mat. Zh. 16 (1964), 61-71.

Department of Mathematical Sciences

Tsinghua University

Beijing 100084

P. R. China

e-mail: gyzhang@mail.tsinghua.edu.cn
Department of Mathematics

Capital Normal University

Beijing 100037

P. R. China

e-mail: qzhli@mail.cnu.edu.cn 